\title{
Racial variations in extracorporeal membrane oxygenation use following congenital heart surgery
}

Titus Chan, MD, MS, MPP, ${ }^{\mathrm{a}}$ Cindy S. Barrett, MD, MPH, ${ }^{\mathrm{b}}$ Yuen Lie Tjoeng, MD, ${ }^{\mathrm{a}}$ Jacob Wilkes, BS,

Susan L. Bratton, MD, MPH, ${ }^{\mathrm{d}}$ and Ravi R. Thiagarajan, MBBS, $\mathrm{MPH}^{\mathrm{e}}$

\section{ABSTRACT}

Objectives: Previous studies demonstrate racial and ethnic disparities among children undergoing congenital heart surgery. Extracorporeal membrane oxygenation (ECMO) is used to support critically ill children after congenital heart surgery and improve survival. Thus, racial or ethnic variations in postoperative ECMO use following congenital heart surgery may be associated with racial/ ethnic disparities in hospital survival.

Methods: All children in the Pediatric Health Information Systems dataset undergoing congenital heart surgery from 2004 to 2015 were examined. Multivariable, multinomial regression models examining hospital survival without ECMO use, survival after ECMO, death after ECMO, and death without ECMO support were constructed.

Results: Of 130,860 congenital cardiac surgery patients, $95.4 \%$ survived to hospital discharge without requiring ECMO support, whereas 1.3\% survived after ECMO support, $1.3 \%$ died after ECMO support, and $1.9 \%$ died without receiving ECMO support. After adjustment for other covariates, black patients (odds ratio, $1.22 ; 95 \%$ confidence interval [CI], 1.05-1.42) and patients of other race (odds ratio, $1.36 ; 95 \% \mathrm{CI}, 1.17-1.58$ ) were at increased odds of mortality compared with white patients. In multivariable multinomial models, black patients had increased risk of death without ECMO support (relative risk, 1.31; 95\% CI, 1.11-1.56). Patients of other race (relative risk, 1.37;95\% CI, 1.10-1.69) and governmental insurance (relative risk, 1.24 ; 95\% CI, 1.12-1.37) were also at increased risk of death without ECMO.

Conclusions: Black children and children of other race are at increased odds of mortality after congenital heart surgery. These disparities can be traced to variations in ECMO utilization across racial/ethnic groups. (J Thorac Cardiovasc Surg 2018;156:306-15)

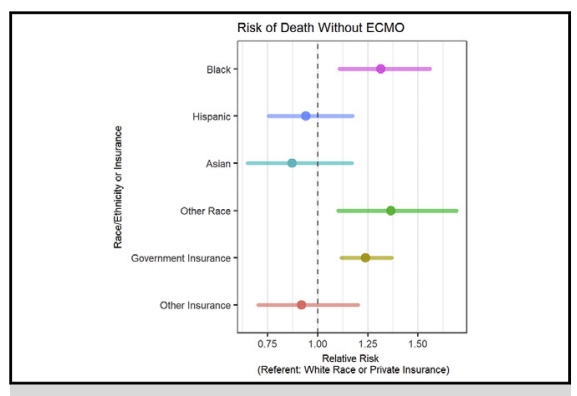

Black race and other nonwhite race as well as government insurance are risk factors for death without ECMO.

\section{Central Message}

Nonwhite and publicly insured children are more likely to die without receiving ECMO after cardiac surgery. This variation in ECMO use contributes to postsurgical mortality.

\section{Perspective}

Nonwhite children and children with government insurance have higher rates of mortality after congenital heart surgery. We demonstrate that race and insurance are risk factors for death without ECMO and may be the driver of postsurgical disparities. Changing how ECMO is discussed and offered to patients' families may be the first step in improving differences in surgical mortality.

See Editorial Commentary page 316.

See Editorial page 291.

\footnotetext{
From the ${ }^{\mathrm{a} D i v i s i o n}$ of Pediatric Critical Care Medicine and The Heart Center, Seattle Children's Hospital, University of Washington, Seattle, Wash; ' Department of Cardiology, Children's Hospital Colorado, University of Colorado School of Medicine, Aurora, Colo; 'Quality and Informatics, Intermountain Healthcare, Salt Lake City, Utah; ${ }^{\mathrm{d}}$ Pediatric Critical Care Medicine, University of Utah, Salt Lake City, Utah; and 'Department of Cardiology, Children's Hospital Boston and Harvard Medical School, Boston, Mass.

Received for publication Aug 29, 2017; revisions received Feb 9, 2018; accepted for publication Feb 15, 2018; available ahead of print April 19, 2018.

Address for reprints: Titus Chan, MD, MS, MPP, Division of Pediatric Critical Care Medicine and The Heart Center, Seattle Children's Hospital, 4800 Sand Point Way, NE, M/S: FA.2.112, Seattle, WA 98105 (E-mail: titus.chan@seattlechildrens.org). $0022-5223 / \$ 36.00$

Copyright (c) 2018 by The American Association for Thoracic Surgery https://doi.org/10.1016/j.jtcvs.2018.02.103
}

Nonwhite children are at increased risk of mortality following cardiac surgery. ${ }^{1-4}$ The etiology behind postsurgical survival disparities in this population is

- Scanning this $\mathrm{QR}$ code will take you to a supplemental video for the article. 


\section{Abbreviations and Acronyms \\ CCC = complex chronic conditions \\ $\mathrm{CPR}=$ cardiopulmonary resuscitation \\ CTC = clinical transaction code \\ ECMO $=$ extracorporeal membrane oxygenation \\ ICD-9-CM = International Classification of Diseases, Ninth Revision, Clinical Modification \\ IVH = intraventricular hemorrhage \\ PHIS $=$ Pediatric Health Information System \\ RACHS $=$ Risk Adjustment for Congenital Heart Surgery}

unclear. Previous studies show that nonwhite patients are at increased risk of experiencing a complication ${ }^{3}$ and failureto-rescue ${ }^{5}$ (mortality after experiencing a complication). However, previous studies of failure-to-rescue included extracorporeal membrane oxygenation (ECMO) as a postoperative complication and not as a support modality.

Although requiring ECMO is an undesired outcome, ECMO is used to improve survival of cardiac surgical patients who would have otherwise likely died. ${ }^{6}$ Thus, variations in ECMO use across populations may result in differences in overall postsurgical survival. If ECMO use varies across race or ethnicity, this may contribute to racial or ethnic disparities in hospital mortality. For these reasons, we examined an administrative database of children's hospitals, to assess variations in ECMO use across racial or ethnic groups in children undergoing congenital heart surgery. Furthermore, we also evaluated the influence of ECMO use on overall hospital survival.

\section{PATIENTS AND METHODS}

This was a retrospective, cross-sectional study using the Pediatric Health Information System (PHIS) database, an administrative dataset maintained by the Children's Hospital Association. PHIS includes administrative and billing data from 49 tertiary care children's hospitals. Participating hospitals provide information on demographic characteristics, outcomes, diagnoses, procedures, and charge codes. The majority of hospitals also submit data for pharmacy, medical imaging, laboratory, supply, nursing, and therapy services using clinical transaction classification (CTC) codes for billed services. Use of de-identified administrative data qualified for exemption from human subjects review by the University of Utah Institutional Review Board.

We included all patients aged $\leq 18$ years discharged from a participating children's hospital from January 1, 2004, to September 30, 2015, with a cardiac surgical procedure. PHIS uses International Classification of Diseases, Ninth Revision, Clinical Modification (ICD-9-CM) procedure and diagnostic codes to assign Risk Adjustment for Congenital Heart Surgery (RACHS) categories to all qualifying patients. ${ }^{7}$ All patients with either a RACHS category or procedure codes consistent with a cardiac surgical procedure (eg, cardiopulmonary bypass, hypothermia related to cardiac surgery, cardioplegia, intraoperative pacemaker, or shunts that may be performed off bypass) were included for analysis. Postoperative ECMO was identified using ICD-9-CM and CTC codes. ICD-9-CM diagnosis codes are recorded for the entire hospital stay, whereas CTC and procedure codes are reported with a date of service, enabling investigators to establish a chronology of procedures but not diagnoses.

Because this study focused on variations in postoperative ECMO use, patients who underwent ECMO before cardiac surgery $(n=628)$ or those in which the timing of surgery could not be established ( $\mathrm{n}=5669$ with 45 cases also undergoing ECMO support) were excluded. Patients were grouped into 4 mutually exclusive discharge outcomes: survival without ECMO, survival after ECMO support, death after or during ECMO support, and death without ECMO support. Overall or hospital mortality includes patients who died after ECMO and those who died without ECMO support.

The independent variable of interest was race and ethnicity. Over the study period, PHIS used 2 different systems of classifying race and ethnicity. The first system (used until 2009) classified race as 1 of 6 mutually exclusive categories (white, black, Asian, American Indian, other, and missing race) and ethnicity as either Hispanic or not Hispanic/unknown. The second system (used 2007 onward) classified each racial group (white, black, Asian, Pacific Islander, American Indian, and other) as a dichotomous variable such that patients could have multiple race categories. Ethnicity was classified as Hispanic, not Hispanic, or unknown. For this analysis, patients were categorized as non-Hispanic white (hereafter referred to as white), non-Hispanic black (hereafter referred to as black), Hispanic ethnicity (including all patients with Hispanic ethnicity, regardless of race), Asian/Pacific Islander (hereafter referred to as Asian), and other race (including multirace). Patients with unknown ethnicity were categorized as non-Hispanic. Primary payer was categorized as private insurance, government payer, and other payer.

Age at the time of surgical procedure was categorized as $<29$ days, 1 month to 1 year, 1 to 4 years, 5 to 12 years, and older than age 12 years. Surgical complexity was grouped into low (RACHS 1 and 2), medium (RACHS 3 and 4), and high complexity (RACHS 5 and 6) procedures or no RACHS category. Using ICD-9-CM diagnosis codes, we examined other covariates that may influence outcomes and decisions to utilize ECMO for postsurgical cardiac support. Complex chronic conditions (CCCs) as defined by Feudtner and colleagues ${ }^{8}$ were analyzed for each noncardiac organ system. The total number of noncardiac organ system CCCs were totaled and categorized as 0,1 , or $\geq 2$ organ systems as a measure of underlying patient complexity. Patients born at $<37$ weeks' gestational age were categorized as born prematurely. Using CTC codes, presurgical mechanical ventilation and preoperative medications were used as a marker of severity of illness. Preoperative administration of vasoactive medication infusions, antiarrhythmic agents, beta-receptor antagonists (ie, beta-blockers), and pulmonary vasodilators before surgery were included as dichotomous variables. Performance of cardiopulmonary resuscitation (CPR) before and after surgical intervention was assessed. Because billing data (CTC codes) were used to identify therapies used during surgical admissions, hospitals without any billing data were excluded from the analysis.

Patient characteristics are presented as percentages for categorical variables. We examined baseline characteristics by race or ethnicity and performed a univariate analysis of predictors of outcome. Differences in predictors of outcome were analyzed using the $\chi^{2}$ test and variables found to be statistically significant $(P<.05)$ were considered for inclusion into the multivariable analysis. We constructed multiple multivariable regression models that adjusted for sex, age, payer group, surgical complexity, surgical era, hospital surgical volume, prematurity, number of CCCs, presurgical mechanical ventilation, preoperative administration of vasoactive infusions, antiarrhythmic agents, beta-blockers, pulmonary vasodilators, preoperative CPR, and postoperative CPR to examine the relationship between race or ethnicity and outcomes after cardiac surgery. Given the relatively short duration of postoperative hospitalizations, the lack of granularity in regard to time measurement in PHIS and the conditional 
nature of some of the outcomes, logistic and multinomial regression models were used to analyze these outcomes. First, we performed a logistic regression model examining overall hospital mortality that accounted for clustering of patient outcomes among hospitals (overall mortality, basic model). To examine within-hospital differences by race or ethnicity (ie, a model that examines whether patients of different race or ethnicity receive different care in the same hospital), we performed a fixed-effects logistic regression model for overall hospital mortality (overall mortality, within hospital). In a similar manner, we constructed a general logistic and a fixed-effects logistic regression model that examined death without ECMO. Additionally, we constructed a multivariable multinomial model that examined the 4 possible clinical outcomes: survival without ECMO (the baseline outcome); survival after ECMO support; death after ECMO support; and death without ECMO support, including the aforementioned covariates. We tested for interactions between race and ethnicity and insurance status.

Because the temporal relationship of diagnoses cannot be constructed from administrative datasets, it is not possible to determine whether certain diagnoses influenced decisions to use ECMO or were a result of ECMO therapy. For example, intraventricular hemorrhage (IVH) could be both a deterrent to use ECMO and an adverse outcome during ECMO support. For this reason, certain ICD-9-CM diagnoses (eg, IVH, intracranial hemorrhage, cerebral thromboembolism, seizures, renal or liver failure, pulmonary or gastrointestinal hemorrhage, sepsis, or postoperative renal support) were not included in the main multivariable analysis. However, these variables were compared among patients that died without ECMO support. Additionally, in a sensitivity analysis, we added these previously omitted covariates into the multinomial model to determine whether these covariates would significantly alter the association between race or ethnicity and outcomes. Secondly, because race or ethnicity is associated with many of the covariates that are associated with the outcomes, we performed a propensity-weighted analysis using the covariates from the mortality logistic regression model. Patients were assigned a weight proportional to the probability that the abovementioned covariates matched patients of white race. This weighting was then used in a multinomial regression model examining the association of race or ethnicity with the 4 outcomes described above. Thirdly, we constructed a competing risk regression model to examine the competing outcomes of survival without ECMO, ECMO support (including death and survival after ECMO), or death without ECMO. Finally, we examined the race or ethnicity composition of each hospital across the 2 race or ethnicity classification systems. Hospitals $(n=6)$ that had high rates of missing, unknown, or other race or ethnicity data were excluded ( $n=16,801$ patients) and the previously described regression models were reconstructed.

\section{RESULTS}

Among 130,860 patients undergoing congenital heart surgery at 48 centers, the majority were of white race $(52.1 \%)$, with black $(12.5 \%)$, Hispanic $(18.6 \%)$, and Asian $(3.5 \%)$ comprising the other identifiable race or ethnic groups. The baseline characteristics by patient race or ethnicity are listed in Table 1. Across all race or ethnicity groups, there was significant variation of sex, age, and payer groups; surgical complexity; prematurity; number of CCCs; surgical era; preoperative use of mechanical ventilation; preoperative use of vasoactive medication infusions; antiarrhythmic medications; beta-blockers; pulmonary vasodilators; preoperative CPR; postoperative CPR; and clinical outcome (all $P$ values $<.01$ ).

When race or ethnicity was dichotomized into white versus nonwhite categories, nonwhite patients were more likely to be female, younger, receive government insurance, born prematurely, and have at least $1 \mathrm{CCC}$ (all $P$ values $<.01$ ). Compared with white patients, nonwhite patients were also more likely to receive preoperative mechanical ventilation, vasoactive infusions, antiarrhythmic agents, beta-blockers, pulmonary vasodilators, preoperative CPR, and postoperative CPR (all $P$ values $<.01$ ). Although nonwhite patients had similar rates of ECMO use (white, $2.6 \%$ vs nonwhite, $2.7 \%$ ), nonwhite patients were significantly less likely to survive without ECMO $(95.9 \%$ vs $95.1 \%$ ) and were significantly more likely to die without ECMO support $(1.5 \%$ vs $2.2 \%)(P<.01)$.

Table 2 lists clinical characteristics of patients by their clinical outcome. Most patients undergoing cardiac surgery survived to hospital discharge without ECMO (95.4\%), whereas $1.3 \%$ survived after ECMO support, $1.3 \%$ died after ECMO support, and $1.9 \%$ died without ECMO support. There was statistically significant variation in distribution of sex, age, and payer groups, surgical complexity, prematurity, number of CCCs, surgical era, presurgical mechanical ventilation, preoperative administration of vasoactive medication infusions, antiarrhythmic agents, betablockers, pulmonary vasodilators, preoperative CPR, and postoperative CPR across clinical outcome categories (all $P$ values $<.01$ ). Hospital mortality was significantly associated with younger age, government insurance, higher surgical complexity, prematurity, presence of CCCs, earlier surgical era, preoperative mechanical ventilation, preoperative receipt of vasoactive infusions, antiarrhythmic agents, beta-blockers, pulmonary vasodilators, preoperative CPR, and postoperative CPR (all $P$ values $<.01$ ).

Multivariable models evaluating the association of race or ethnicity and hospital mortality, adjusted for the previously described covariates, are shown in Figure 1. Black patients (odds ratio [OR], 1.22; 95\% confidence interval [CI], 1.05-1.42), patients of other race (OR, 1.36; 95\% CI, 1.17-1.58), and patients with government insurance (OR, $1.21 ; 95 \%$ CI, 1.10-1.32) had increased odds of mortality. A second fixed-effects logistic regression model of withinhospital effects (Figure 1) continued to demonstrate increased odds of mortality for patients of black race (OR, $1.19 ; 95 \%$ CI, 1.07-1.32), patients of other race (OR, 1.43; $95 \%$ CI, 1.28-1.60), and patients with governmentprovided insurance (OR, 1.17; 95\% CI, 1.08-1.27).

In a multivariable logistic regression model for death without ECMO support using the same covariates, black race (OR, 1.31; 95\% CI, 1.11-1.55), other race (OR, 1.35; 95\% CI, 1.08-1.68), and government insurance (OR, $1.23 ; 95 \%$ CI, 1.12-1.36) showed increased odds of dying without ECMO support (Figure 1). This association remained in fixed-effects models that examined withinhospital effects (Figure 1).

We examined the association of race or ethnicity with the multiple possible outcomes after cardiac surgery in a multinomial regression model using the same covariates as the 
TABLE 1. Characteristics by race or ethnicity

\begin{tabular}{|c|c|c|c|c|c|c|}
\hline & $\begin{array}{c}\text { White } \\
\mathrm{n}=\mathbf{6 8 , 1 5 5 , 5 2 . 1 \%}\end{array}$ & 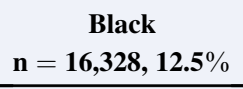 & $\begin{array}{c}\text { Hispanic } \\
\mathbf{n}=\mathbf{2 4 , 3 8 7 , 1 8 . 6 \%}\end{array}$ & $\begin{array}{c}\text { Asian } \\
\mathrm{n}=\mathbf{4 6 0 7 , 3 . 5 \%} \\
\end{array}$ & $\begin{array}{c}\text { Other } \\
\mathrm{n}=\mathbf{1 2 , 7 5 2 , 9 . 7 \%} \\
\end{array}$ & $\begin{array}{c}\text { Missing } \\
\mathrm{n}=\mathbf{4 6 3 1}, \mathbf{3 . 5} \%\end{array}$ \\
\hline Male & $30,033(44)$ & 7639 (47) & $10,942(45)$ & $2121(46)$ & $5728(45)$ & $2086(45)$ \\
\hline \multicolumn{7}{|l|}{ Age group } \\
\hline$<29 \mathrm{~d}$ & $15,245(22)$ & $3290(20)$ & $5912(24)$ & 897 (19) & $3533(28)$ & $1424(31)$ \\
\hline $1 \mathrm{mo}-1 \mathrm{y}$ & $24,846(36)$ & $6506(40)$ & $9311(38)$ & $1652(36)$ & 4958 (39) & $1660(36)$ \\
\hline $1 y-4 y$ & $14,772(22)$ & $3419(21)$ & $5120(21)$ & $1126(24)$ & $2350(18)$ & 790 (17) \\
\hline $5-12 y$ & $7412(11)$ & $1825(11)$ & $2629(11)$ & $597(13)$ & $1231(10)$ & $463(10)$ \\
\hline$>12 \mathrm{y}$ & $5880(9)$ & $1288(8)$ & $1415(6)$ & $335(7)$ & $680(5)$ & $294(6)$ \\
\hline \multicolumn{7}{|l|}{ Insurance } \\
\hline Private & $35,490(52)$ & $3370(21)$ & 4273 (18) & $2177(47)$ & $4114(32)$ & $1862(40)$ \\
\hline Government & $23,867(35)$ & $11,302(69)$ & $17,632(72)$ & $1744(38)$ & $6424(50)$ & $2168(47)$ \\
\hline \multicolumn{7}{|l|}{ Era } \\
\hline 2004-2007 & $21,382(31)$ & $5262(32)$ & 7843 (32) & $1143(25)$ & 4349 (34) & $1504(32)$ \\
\hline $2008-2010$ & $17,988(26)$ & $4269(26)$ & $6633(27)$ & $1103(24)$ & $3252(26)$ & 797 (17) \\
\hline 2011-2015 & $28,785(42)$ & $6797(42)$ & $9911(41)$ & $2361(51)$ & $5151(40)$ & $2330(50)$ \\
\hline \multicolumn{7}{|l|}{ Surgical complexity } \\
\hline RACHS 1 and 2 & $27,539(40)$ & $6513(40)$ & $9899(41)$ & 2039 (44) & $5275(41)$ & $1901(41)$ \\
\hline RACHS 3 and 4 & $30,182(44)$ & $6738(41)$ & $10,533(43)$ & $1909(41)$ & $5401(42)$ & $2012(43)$ \\
\hline RACHS 5 and 6 & $2167(3)$ & $466(3)$ & $855(3)$ & $99(2)$ & $419(3)$ & $163(4)$ \\
\hline No RACHS category & 8267 (12) & $2611(16)$ & $3100(13)$ & $560(12)$ & $1657(13)$ & $555(12)$ \\
\hline Prematurity & $3665(5)$ & $1521(9)$ & $1563(6)$ & $246(5)$ & $1015(8)$ & $420(9)$ \\
\hline \multicolumn{7}{|l|}{ Number of CCCs } \\
\hline 1 & $15,609(23)$ & 3921 (24) & $6162(25)$ & 919 (20) & 2937 (23) & $1031(22)$ \\
\hline 2 or more & 7875 (12) & $2146(13)$ & 3497 (14) & $552(12)$ & $1483(12)$ & $572(12)$ \\
\hline \multicolumn{7}{|l|}{ Preoperative therapy } \\
\hline Mechanical ventilation & $9984(15)$ & 2987 (18) & $4020(16)$ & $664(14)$ & $2299(18)$ & $1043(23)$ \\
\hline CPR & $1074(2)$ & $261(2)$ & $318(1)$ & $88(2)$ & $462(4)$ & $81(2)$ \\
\hline Vasoactive infusion & $11,483(17)$ & $3509(21)$ & $4975(20)$ & $870(19)$ & $2640(21)$ & $1003(22)$ \\
\hline Antiarrhythmic & $3325(5)$ & $1057(6)$ & $1426(6)$ & $224(5)$ & $813(6)$ & $244(5)$ \\
\hline Beta antagonist & $1450(2)$ & $485(3)$ & $611(3)$ & $105(2)$ & $258(2)$ & $91(2)$ \\
\hline Pulmonary vasodilator & $1322(2)$ & $436(3)$ & $510(2)$ & $108(2)$ & $281(2)$ & $140(3)$ \\
\hline Postoperative CPR & $4228(6)$ & $1038(6)$ & $2480(10)$ & $377(8)$ & $1063(8)$ & $208(4)$ \\
\hline \multicolumn{7}{|l|}{ Discharge status } \\
\hline Survived without ECMO & $65,348(95.9)$ & $15,458(94.7)$ & $23,291(95.5)$ & $4428(96.1)$ & $12,038(94.4)$ & $4302(92.9)$ \\
\hline Survived after ECMO & $931(1.4)$ & 237 (1.4) & $298(1.2)$ & $54(1.2)$ & $166(1.3)$ & $55(1.2)$ \\
\hline Died after ECMO & $818(1.2)$ & $221(1.4)$ & $283(1.2)$ & $60(1.3)$ & $233(1.8)$ & $126(2.7)$ \\
\hline Died without ECMO & $1058(1.5)$ & $412(2.5)$ & $515(2.1)$ & $65(1.4)$ & $315(2.5)$ & $148(3.2)$ \\
\hline
\end{tabular}

Values are presented as $\mathrm{n}(\%) . R A C H S$, Risk Adjustment for Congenital Heart Surgery; $C C C$, complex chronic conditions, $C P R$, cardiopulmonary resuscitation; $E C M O$, extracorporeal membrane oxygenation.

previous logistic regression models. Survival without ECMO was used as the baseline outcome with survival after ECMO, death after ECMO, and death without ECMO support as the alternate outcomes (Figure 2). Compared with patients who survived without ECMO, race or ethnicity was not associated with survival after ECMO. However, patients of other race had increased risk of death after ECMO (relative risk [RR], 1.33; 95\% CI, 1.04-1.70), whereas Hispanic ethnicity was associated with decreased risk of death after ECMO (RR, 0.72; 95\% CI, 0.55-0.95). Government insurance was also associated with increased risk of death after ECMO (RR, 1.18; 95\% CI, 1.02-1.36). When examining death without ECMO, black race (RR, 1.31; 95\%
CI, 1.11-1.56), other race (RR, 1.37; 95\% CI, 1.101.69 ), and government insurance (RR, $1.24 ; 95 \% \mathrm{CI}$, 1.12-1.37) were associated with increased risk of death without ECMO support. There was no significant interaction between race or ethnicity and insurance (data not shown).

As a sensitivity analysis, we performed a univariate analysis of the characteristics of patients who died without ECMO by race or ethnicity (Table 3 ). There were significant differences across all races or ethnicities in regard to sex, age groups $(P=.021)$, insurance status, surgical complexity, prematurity, number of CCCs, era, and preoperative mechanical ventilation $(P<.01$ for all other 
TABLE 2. Characteristics by discharge status and extracorporeal membrane oxygenation (ECMO) use

\begin{tabular}{|c|c|c|c|c|c|}
\hline & $\begin{array}{l}\text { Survived without ECMO } \\
\qquad(\mathrm{n}=\mathbf{1 2 4 , 8 6 5 )}\end{array}$ & $\begin{array}{c}\text { Survived after ECMO } \\
(\mathrm{n}=\mathbf{1 7 4 1})\end{array}$ & $\begin{array}{c}\text { Died after ECMO } \\
\quad(n=1741)\end{array}$ & $\begin{array}{l}\text { Died without ECMO } \\
\quad(n=2513)\end{array}$ & $P$ value \\
\hline Male & $55,856(45)$ & $746(43)$ & $760(44)$ & $1187(47)$ & $<.001$ \\
\hline Age group & & & & & $<.001$ \\
\hline$<29 d$ & $26,861(21)$ & $883(51)$ & $1126(65)$ & $1431(57)$ & \\
\hline $1 \mathrm{mo}-1 \mathrm{y}$ & $47,191(38)$ & $529(30)$ & $417(24)$ & 796 (32) & \\
\hline $1-4 y$ & 27,091 (22) & 207 (12) & $114(6)$ & $165(6)$ & \\
\hline $5-12 y$ & $13,978(11)$ & $74(4)$ & $38(2)$ & $67(3)$ & \\
\hline$>12 \mathrm{y}$ & $9744(8)$ & $48(3)$ & $46(3)$ & $54(2)$ & \\
\hline Insurance & & & & & $<.001$ \\
\hline Private & $49,300(39)$ & $639(37)$ & $590(34)$ & $757(30)$ & \\
\hline Government & $59,864(48)$ & 855 (49) & 925 (53) & $1493(59)$ & \\
\hline Era of surgery & & & & & $<.001$ \\
\hline $2004-2007$ & $39,330(32)$ & $518(30)$ & $590(34)$ & $1045(41)$ & \\
\hline $2008-2010$ & $32,580(26)$ & $426(24)$ & $412(24)$ & $624(25)$ & \\
\hline 2011-2015 & $52,955(42)$ & 797 (46) & $739(42)$ & 844 (34) & \\
\hline Surgical complexity & & & & & $<.001$ \\
\hline RACHS 1 and 2 & $52,409(42)$ & $212(12)$ & $140(8)$ & 405 (16) & \\
\hline RACHS 3 and 4 & $53,605(43)$ & $952(55)$ & $957(55)$ & $1261(50)$ & \\
\hline RACHS 5 and 6 & 3339 (3) & $233(13)$ & $323(19)$ & $274(11)$ & \\
\hline No RACHS category & $15,512(12)$ & $344(20)$ & $321(18)$ & $573(23)$ & \\
\hline Prematurity & $7468(6)$ & $109(6)$ & $210(12)$ & $643(26)$ & $<.001$ \\
\hline Number of CCCs & & & & & $<.001$ \\
\hline 1 & $28,646(23)$ & $547(31)$ & $636(37)$ & $750(30)$ & \\
\hline 2 or more & $14,088(11)$ & $490(28)$ & $534(31)$ & $1013(40)$ & \\
\hline \multicolumn{6}{|l|}{ Preoperative therapy } \\
\hline Mechanical ventilation & $18,375(15)$ & $541(31)$ & $716(41)$ & $1365(54)$ & $<.001$ \\
\hline CPR & 1964 (2) & $79(5)$ & $72(4)$ & $169(7)$ & $<.001$ \\
\hline Vasoactive infusion & $21,790(17)$ & $652(37)$ & $785(45)$ & $1253(50)$ & $<.001$ \\
\hline Antiarrhythmic & $6550(5)$ & $122(7)$ & $158(9)$ & $259(10)$ & $<.001$ \\
\hline Beta antagonist & $2784(2)$ & $60(3)$ & $68(4)$ & $88(4)$ & $<.001$ \\
\hline Pulmonary vasodilator & 2373 (2) & $94(5)$ & $106(6)$ & $224(9)$ & $<.001$ \\
\hline Postoperative CPR & $7652(6)$ & $538(31)$ & $560(32)$ & $644(26)$ & $<.001$ \\
\hline
\end{tabular}

Values are presented as n (\%). ECMO, Extracorporeal membrane oxygenation; $R A C H S$, Risk Adjustment for Congenital Heart Surgery; $C C C$, complex chronic conditions; $C P R$, cardiopulmonary resuscitation.

comparisons). In contrast, there were no significant differences in preoperative treatment with vasoactive medication infusions, antiarrhythmic agents, beta-blockers, pulmonary vasodilators, and preoperative and postoperative CPR (all $P$ values $>.05$ ) across all races or ethnicities in patients who died without ECMO support. When examining factors that may be judged as contraindications to ECMO, IVH $(P=.008)$ and postoperative renal support $(P=.005)$ significantly varied across race or ethnicity in patients who died without ECMO. However, intracranial, pulmonary, or gastrointestinal hemorrhage; seizures; cerebral thromboembolism; sepsis; and liver failure did not differ across race or ethnicity in these patients (all $P$ values $>.05$ ).

A multivariable, multinomial regression model that included the previously omitted covariates such as IVH demonstrated that black infants and children continued to have increased risk of death without ECMO (RR, 1.29; 95\% CI, 1.08-1.53). Likewise, other race (RR, 1.38; 95\% CI, 1.11-1.71) and government insurance (RR, 1.21; 95\% CI, 1.09-1.34) remained risk factors for death without ECMO. Additionally, in these models, Hispanic ethnicity was associated with decreased risk of death after ECMO (RR, 0.67; 95\% CI, 0.46-0.98), whereas other race (RR, $1.39,95 \% \mathrm{CI} ; 1.02-1.37)$ and government insurance (RR, $1.19 ; 95 \% \mathrm{CI}, 1.02-1.37)$ were associated with increased risk of death after ECMO. As part of the sensitivity analysis, we performed a propensity-weighted analysis and a competing risk model as described above. In the propensity-weighted multinomial regression model, black patients (RR, 1.27; 95\% CI, 1.08-1.50) and patients of other race $(\mathrm{RR}, 1.29 ; 95 \% \mathrm{CI}, 1.05-1.59)$ remained at increased 
risk of death without ECMO. Patients of other race (RR, $1.37 ; 95 \%$ CI, 1.04-1.81) were also at increased risk of death after ECMO. In the competing risk model, black race (hazard ratio [HR], 1.30; 95\% CI, 1.12-1.52) and other race (HR, 1.30; 95\% CI, 1.07-1.58) were risk factors for death without ECMO. Black race (HR, 0.90; 95\% CI, 0.84-0.97) was also associated with decreased survival without ECMO. Finally, after excluding hospitals with high rates of missing, unknown, or other race or ethnicity data, black race, other race, and government insurance continued to be significant risk factors for death without ECMO (not shown).

\section{DISCUSSION}

We found that black race, other race, and government insurance were independent risk factors for mortality after congenital heart surgery. Additionally, these children had increased risk of death without ECMO support, whereas other race and government insurance remained independently associated with death after ECMO (Video 1). Furthermore, in black children, racial variation in death without ECMO support may be a strong contributor to postsurgical survival disparities. In contrast, both mortality after ECMO and death without ECMO appear to be contributors to increased postsurgical mortality in children of other race and using government insurance.

Previous studies of adult and pediatric patients reported poorer outcomes in nonwhite patients seeking cardiac care, ${ }^{1-4,9-12}$ likely related to variations in both hospital referral patterns and in-hospital treatment. Studies of acute myocardial infarction care have found both disparities in hospital referral patterns ${ }^{9,10}$ as well as delays and variations in allocation of treatment for black patients. $^{12,13}$ However, studies of congenital surgery patients consistently demonstrate lower survival in black patients that cannot be explained by hospital referral patterns alone. ${ }^{2,5}$

This study provides further insight into the mechanisms behind racial disparities in congenital surgical survival outcomes. Variations in ECMO use appear to be among the drivers of survival disparities in black patients, whereas both increased mortality after ECMO and variations in ECMO use appear to be contributing to higher mortality for patients of other race and government insurance. These differences are not due to hospital referral patterns but appear to be due to variations in within-hospital treatment allocation.

The reasons for the greater rates of death without ECMO among black children, children of other race, and children receiving government insurance are unclear. Multiple factors contribute to medical decision making when considering ECMO support, including prior and current clinical condition, comorbidities, and social/familial factors. Additionally, centers and physicians vary in regard to ECMO use, indications, and contraindications for ECMO support. ${ }^{6}$ Because the PHIS does not contain data regarding the

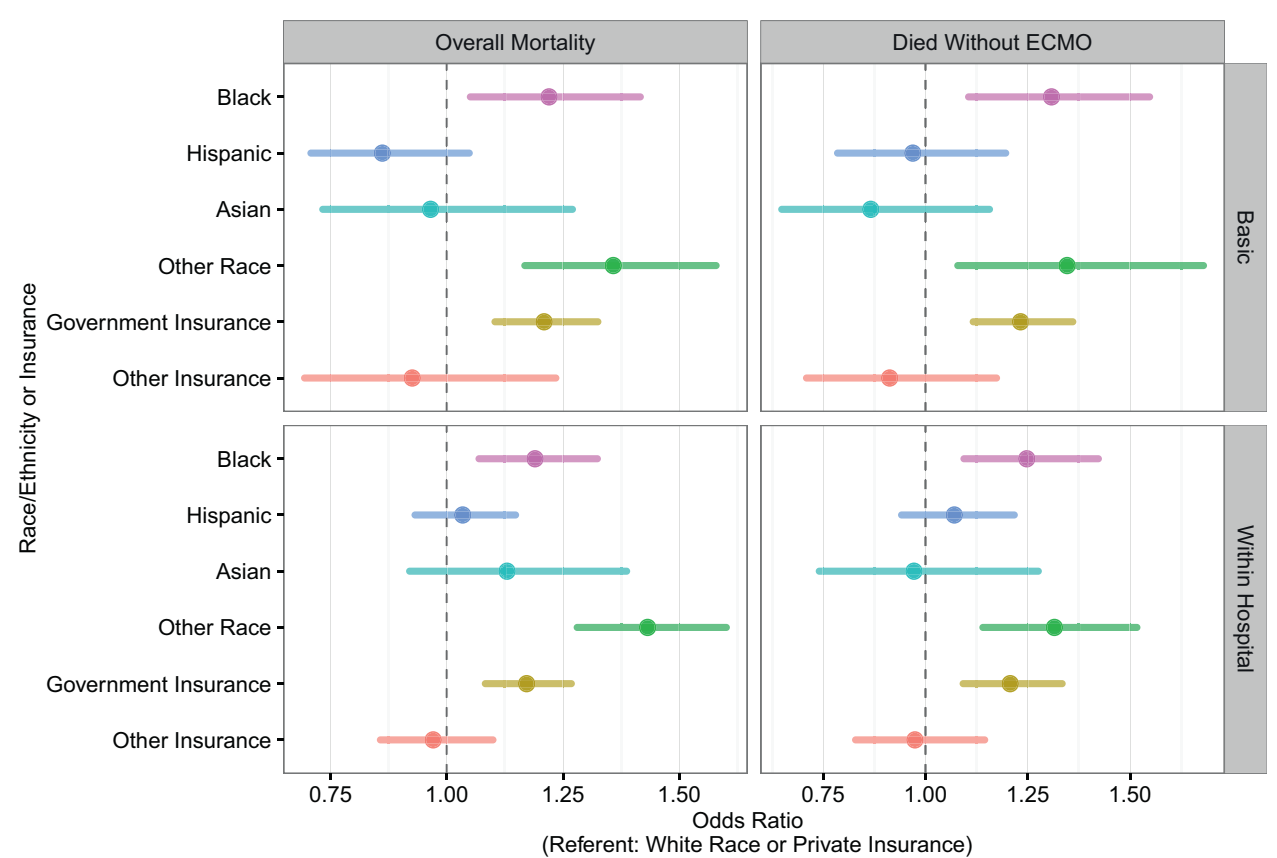

FIGURE 1. Multivariable logistic regression models evaluating the association of race or ethnicity with overall mortality and dying without extracorporeal membrane oxygenation $(E C M O)$. All models adjusted for sex, age, surgical complexity, surgical era, prematurity, complex chronic conditions, hospital surgical volume, preoperative mechanical ventilation and medications, and pre- and postoperative cardiopulmonary resuscitation. 


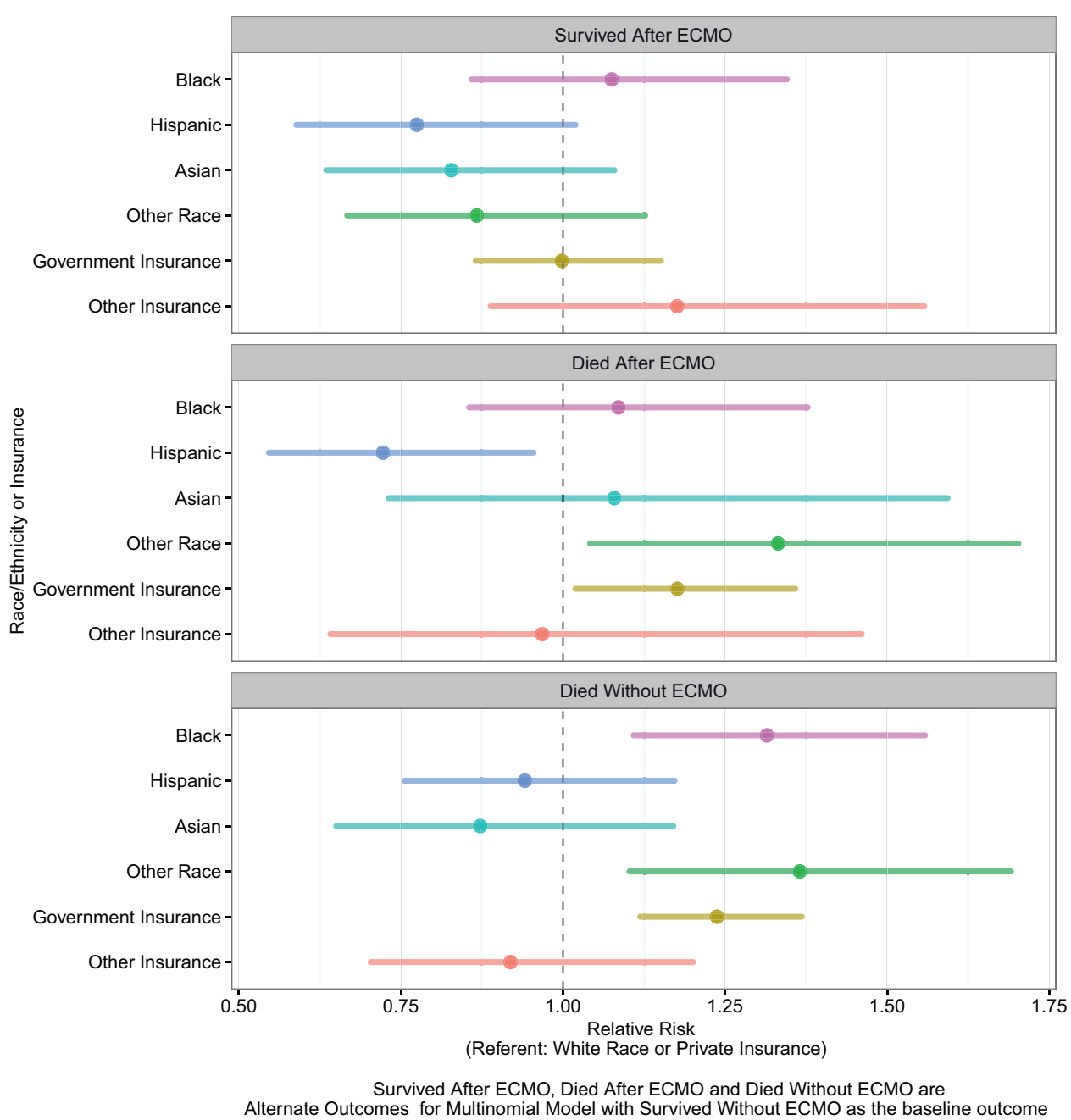

FIGURE 2. Multivariable multinomial models for discharge status and extracorporeal membrane oxygenation (ECMO) use. Survived after ECMO, died after ECMO, and died without ECMO are alternate outcomes for multinomial model with survived without ECMO as the baseline outcome. All models adjusted for sex, age, surgical complexity, surgical era, prematurity, complex, chronic conditions, hospital surgical volume, preoperative mechanical ventilation and medications, and pre- and postoperative cardiopulmonary resuscitation.

decision-making process for ECMO use, we adjusted for circumstances that could influence decisions regarding ECMO use. If race or ethnicity did not influence medical decision making, we would expect similar rates of death without ECMO across all race or ethnicities. However, nonwhite race and government insurance remained risk factors for death without ECMO. Furthermore, regression models that included factors that may be a deterrent to ECMO support (such as diagnoses of central nervous system hemorrhage) did not change the association among race, insurance, and death without ECMO.

This suggests that, although there may be provider and center-level variability in indications for ECMO use, variability in clinical covariates and relative contraindications to ECMO were not important contributors in disparities in ECMO use. Social factors, such as implicit racial biases and cultural preferences, may contribute to disparities in
ECMO use. Previous studies demonstrate that therapeutic decisions by health care professionals can be influenced by implicit bias. ${ }^{14,15}$ Implicit racial and social bias may influence how health care providers present the option to use ECMO to patient families. Conversely, cultural preferences may also influence decisions regarding ECMO use. The PHIS does not contain data regarding family care decisions or directives. Thus, it is possible that patients died without ECMO after parents declined this therapy. Studies of adult patients ${ }^{16,17}$ suggest that nonwhite patients are more likely to desire and undergo high-intensity therapies, suggesting that cultural preferences for avoiding highly invasive therapy may not be a contributor to disparities in ECMO use.

We did not find an association between black race and mortality in patients receiving ECMO support. Prior studies that examined different ECMO populations demonstrated 
TABLE 3. Characteristics of patients who died without extracorporeal membrane oxygenation (ECMO), by race or ethnicity

\begin{tabular}{|c|c|c|c|c|c|c|c|}
\hline & White $(n=1058)$ & Black $(n=412)$ & Hispanic $(n=515)$ & $\operatorname{Asian}(n=65)$ & Other $(n=315)$ & Missing $(n=148)$ & $P$ value \\
\hline Male & $490(46)$ & $190(46)$ & $258(50)$ & $34(52)$ & $150(48)$ & $65(44)$ & .008 \\
\hline Age group & & & & & & & .021 \\
\hline$<29 d$ & $581(55)$ & $202(49)$ & $313(61)$ & $39(60)$ & $201(64)$ & $95(64)$ & \\
\hline $1 \mathrm{mo}-1 \mathrm{y}$ & $346(32)$ & $155(38)$ & $144(28)$ & $17(26)$ & $94(30)$ & $40(27)$ & \\
\hline $1-4 \mathrm{y}$ & $72(7)$ & $32(8)$ & $34(7)$ & $5(8)$ & $13(4)$ & $9(6)$ & \\
\hline $5-12 y$ & $30(3)$ & $14(3)$ & $12(2)$ & $3(5)$ & $6(2)$ & $2(1)$ & \\
\hline$>12 \mathrm{y}$ & $29(3)$ & $9(2)$ & $12(2)$ & $1(1)$ & $1(0)$ & $2(1)$ & \\
\hline Insurance & & & & & & & $<.001$ \\
\hline Private & $450(43)$ & $61(15)$ & $70(14)$ & $27(42)$ & $101(32)$ & $48(32)$ & \\
\hline Government & $483(46)$ & $315(76)$ & $398(77)$ & $32(49)$ & $186(59)$ & $79(53)$ & \\
\hline Era & & & & & & & .001 \\
\hline 2004-2007 & $431(41)$ & $153(37)$ & $221(43)$ & $20(31)$ & $161(51)$ & $59(40)$ & \\
\hline $2008-2010$ & $254(24)$ & $118(29)$ & $140(27)$ & $17(26)$ & $68(22)$ & $27(18)$ & \\
\hline 2011-2015 & $373(35)$ & $141(34)$ & $154(30)$ & $28(43)$ & $86(27)$ & $62(42)$ & \\
\hline Surgical complexity & & & & & & & $<.001$ \\
\hline RACHS 1 and 2 & $178(17)$ & $69(17)$ & $70(14)$ & $10(15)$ & $51(16)$ & $27(18)$ & \\
\hline RACHS 3 and 4 & $535(51)$ & $171(41)$ & $300(58)$ & $30(46)$ & $162(51)$ & $63(43)$ & \\
\hline RACHS 5 and 6 & $118(11)$ & $33(8)$ & $60(12)$ & $4(6)$ & $44(14)$ & $15(10)$ & \\
\hline No RACHS category & $227(21)$ & $139(34)$ & $85(16)$ & $21(32)$ & $58(18)$ & $43(29)$ & \\
\hline Prematurity & $252(24)$ & $146(35)$ & $100(19)$ & $21(32)$ & $80(25)$ & $44(30)$ & $<.001$ \\
\hline Number of CCCs & & & & & & & .001 \\
\hline 1 & $323(31)$ & $130(32)$ & 147 (29) & $14(22)$ & $95(30)$ & $41(28)$ & \\
\hline 2 or more & $398(38)$ & $167(41)$ & $241(47)$ & $35(54)$ & $106(34)$ & $66(45)$ & \\
\hline \multicolumn{8}{|l|}{ Preoperative therapies } \\
\hline Mechanical ventilation & $547(52)$ & $251(61)$ & $269(52)$ & $39(60)$ & $167(53)$ & $92(62)$ & .007 \\
\hline Vasoactive infusions & $520(49)$ & $201(49)$ & $263(51)$ & $35(54)$ & $149(47)$ & $85(57)$ & .38 \\
\hline Antiarrhythmic & $112(11)$ & $48(12)$ & $45(9)$ & $8(12)$ & $29(9)$ & $17(11)$ & .67 \\
\hline Beta antagonist & $36(3)$ & $19(5)$ & $17(3)$ & $3(5)$ & $8(3)$ & $5(3)$ & .75 \\
\hline Pulmonary vasodilator & $83(8)$ & $38(9)$ & $45(9)$ & $9(14)$ & $27(9)$ & $22(15)$ & .08 \\
\hline CPR & $61(6)$ & $29(7)$ & $33(6)$ & $5(8)$ & $29(9)$ & $12(8)$ & .38 \\
\hline Postoperative renal support & $181(17)$ & $57(14)$ & 89 (17) & $9(14)$ & $58(18)$ & $25(17)$ & .005 \\
\hline Postoperative CPR & $260(25)$ & $104(25)$ & $152(30)$ & $19(29)$ & $75(24)$ & $34(23)$ & .281 \\
\hline Intraventricular hemorrhage & $120(11)$ & $68(17)$ & $42(8)$ & $8(12)$ & $37(12)$ & $17(11)$ & .008 \\
\hline Intracranial hemorrhage & $57(5)$ & $15(4)$ & $26(5)$ & $3(5)$ & $9(3)$ & $7(5)$ & .45 \\
\hline Seizures & $141(13)$ & $49(12)$ & $62(12)$ & $11(17)$ & $26(8)$ & $14(9)$ & .14 \\
\hline Cerebral thromboembolism & $80(8)$ & $21(5)$ & $42(8)$ & $4(6)$ & $18(6)$ & $11(7)$ & .44 \\
\hline Pulmonary hemorrhage & $17(2)$ & $11(3)$ & $10(2)$ & $3(5)$ & $8(3)$ & $5(3)$ & .38 \\
\hline Gastrointestinal Hemorrhage & $44(4)$ & $11(3)$ & $23(4)$ & $1(2)$ & $6(2)$ & $6(4)$ & .26 \\
\hline Sepsis & $397(38)$ & $169(41)$ & $212(41)$ & $35(54)$ & $118(37)$ & $63(43)$ & .09 \\
\hline Liver failure & $37(3)$ & $11(3)$ & $25(5)$ & $4(6)$ & $16(5)$ & $5(3)$ & .36 \\
\hline
\end{tabular}

Values are presented as n (\%). RACHS, Risk Adjustment for Congenital Heart Surgery; $C C C$, complex chronic conditions; CPR, cardiopulmonary resuscitation.

increased odds of mortality for nonwhite patients. ${ }^{18-21}$ Additionally, previous studies of limited subgroups of congenital cardiac surgical patients demonstrated an increase in mortality for black patients receiving ECMO. ${ }^{22,23}$ These differences in findings may be due to variations in patient population and covariates between this and other studies. We also found that patients of Hispanic ethnicity were at decreased risk of mortality after ECMO, a novel finding because few ECMO studies have specifically examined Hispanic ethnicity. One previous study of all children receiving ECMO cardiac support (including nonsurgical patients) found that Hispanic children had increased risk of ECMO mortality. ${ }^{21}$ Currently, the reasons for decreased ECMO mortality among Hispanic patients is unclear. Adult studies suggest a Hispanic paradox, where Hispanic patients have 


\section{Racial Variations in Extracorporeal Membrane Oxygenation Use Following Congenital Heart Surgery}

Titus Chan, MD,MS,MPP; Cindy S Barrett, MD, MPH; Yuen Lie Tjoeng, MD; Jacob Wilkes, BS; Susan L. Bratton, MD, MPH; Ravi R. Thiagarajan, MBBS, MPH

VIDEO 1. The lead author discusses the key findings of the study. Video available at: http://www.jtcvsonline.org.

better outcomes than their white counterparts. ${ }^{24,25}$ However, in congenital heart surgery, Hispanic ethnicity has not been consistently associated with survival outcomes. $^{1-4,26-28}$

This study is subject to limitations, largely related to the use of observational data. As with all retrospective analyses, there is a risk of misclassification of data. Race and ethnicity are intricately intertwined with other aspects of socioeconomic status and cannot be understood in isolation. PHIS does not include patient-level socioeconomic data such as household income or parental education and geographic information was not routinely included at the time of data acquisition. For this reason, we included primary insurance as a proxy for these socioeconomic factors. However, if other socioeconomic factors are associated with outcomes, they are likely to be mediators of the effect of race or ethnicity. For example, if adjustment for education weakens the association between race or ethnicity and outcome, this suggests that black patients experience surgical disparities due to underlying social (educational and economic) disparities. Previous longitudinal studies of children with congenital heart disease demonstrate the persistence of racial or ethnic survival disparities after adjustment for other measures of socioeconomic status. ${ }^{29-31}$ Previous studies demonstrated that the RACHS classification system does not fully capture all congenital surgical procedures. ${ }^{32}$ For this reason, we included cardiac surgical procedures that were not included in the RACHS algorithm. Nonetheless, the application of the RACHS methodology to administrative datasets, when compared with clinical datasets, does not result in statistically significantly biased estimates of overall case mix or in-hospital mortality. ${ }^{32,33}$ Finally, because the PHIS dataset is limited to administrative data, clinical factors, such as physiologic measures of severity of illness, which may influence outcomes, cannot be assessed. As a corollary, this study documented variations in ECMO use across race or ethnic groups and from this, we have inferred that treatment allocation disparities exist across these populations. However, the reasons behind this treatment allocation disparity such as provider reluctance to recommend ECMO or familial reluctance to consent to ECMO support remain undetermined.

\section{CONCLUSIONS}

Black children, children of other race, and patients receiving government insurance have increased risk of dying without ECMO support after congenital cardiac surgery. Furthermore, this variation in ECMO use appears to be a major contributor to racial and social survival disparities in this population. Further research to determine reasons behind this variation in ECMO use and its influence are warranted.

\section{Conflict of Interest Statement}

Dr Thiagarajan's institution has received funding to serve on event adjudication committees from Bristol Myers Squibb and Pfizer. All other authors have nothing to disclose with regard to commercial support.

\section{References}

1. Benavidez OJ, Gauvreau K, Jenkins KJ. Racial and ethnic disparities in mortality following congenital heart surgery. Pediatr Cardiol. 2006;27:321-8.

2. Oster ME, Strickland MJ, Mahle WT. Racial and ethnic disparities in postoperative mortality following congenital heart surgery. J Pediatr. 2011;159: 222-6.

3. DiBardino DJ, Pasquali SK, Hirsch JC, Benjamin DK, Kleeman KC, Salazar JD, et al. Effect of sex and race on outcome in patients undergoing congenital heart surgery: an analysis of the Society of Thoracic Surgeons congenital heart surgery database. Ann Thorac Surg. 2012;94:2054-9.

4. Chan T, Pinto NM, Bratton SL. Racial and insurance disparities in hospital mortality for children undergoing congenital heart surgery. Pediatr Cardiol. 2012;33: 1026-39.

5. Chan T, Lion KC, Mangione-Smith R. Racial disparities in failure-to-rescue among children undergoing congenital heart surgery. J Pediatr. 2015;166:812-8.

6. Bratton SL, Chan T, Barrett CS, Wilkes J, Ibsen LM, Thiagarajan RR. Metrics to assess extracorporeal membrane oxygenation utilization in pediatric cardiac surgery programs. Pediatr Crit Care Med. 2017;18:779-86.

7. Jenkins KJ, Gauvreau K, Newburger JW, Spray TL, Moller JH, Iezzoni LI. Consensus-based method for risk adjustment for surgery for congenital heart disease. J Thorac Cardiovasc Surg. 2002;123:110-8.

8. Feudtner C, Feinstein JA, Zhong W, Hall M, Dai D. Pediatric complex chronic conditions classification system version 2: updated for ICD-10 and complex medical technology dependence and transplantation. BMC pediatrics. $2014 ; 14$ : 199.

9. Barnato AE, Lucas FL, Staiger D, Wennberg DE, Chandra A. Hospital-level racial disparities in acute myocardial infarction treatment and outcomes. Med Care. 2005;43:308-19.

10. Bao Y, Kamble S. Geographical distribution of surgical capabilities and disparities in the use of high-volume providers: the case of coronary artery bypass graft. Med Care. 2009; 47:794-802.

11. Becker ER, Rahimi A. Disparities in race/ethnicity and gender in in-hospital mortality rates for coronary artery bypass surgery patients. J Natl Med Assoc. 2006;98:1729-39

12. Bradley EH, Herrin J, Wang Y, McNamara RL, Webster TR, Magid DJ, et al Racial and ethnic differences in time to acute reperfusion therapy for patients hospitalized with myocardial infarction. JAMA. 2004;292:1563-72.

13. Sonel AF, Good CB, Mulgund J, Roe MT, Gibler WB, Smith J, et al. Racial variations in treatment and outcomes of black and white patients with high-risk nonST-elevation acute coronary syndromes: insights from CRUSADE (can rapid risk stratification of unstable angina patients suppress adverse outcomes with early implementation of the ACC/AHA guidelines?). Circulation. 2005;111:1225-32.

14. Sabin JA, Greenwald AG. The influence of implicit bias on treatment recommendations for 4 common pediatric conditions: pain, urinary tract infection, attention deficit hyperactivity disorder, and asthma. Am J Public Health. 2012;102:988-95. 
15. Green AR, Carney DR, Pallin DJ, Ngo LH, Raymond KL, Iezzoni LI, et al. Implicit bias among physicians and its prediction of thrombolysis decisions for black and white patients. J Gen Intern Med. 2007;22:1231-8.

16. Blackhall LJ, Frank G, Murphy ST, Michel V, Palmer JM, Azen SP. Ethnicity and attitudes towards life-sustaining technology. Soc Sci Med. 1999;48: 1779-89.

17. Smith AK, Earle CC, McCarthy EP. Racial and ethnic differences in end-of-life care in fee-for-service Medicare beneficiaries with advanced cancer. J Am Geriatr Soc. 2009;57:153-8.

18. Chan T, Thiagarajan RR, Frank D, Bratton SL. Survival after extracorporeal cardiopulmonary resuscitation in infants and children with heart disease. J Thorac Cardiovasc Surg. 2008;136:984-92.

19. Thiagarajan RR, Laussen PC, Rycus PT, Bartlett RH, Bratton SL. Extracorporeal membrane oxygenation to aid cardiopulmonary resuscitation in infants and children. Circulation. 2007;116:1693-700.

20. Brogan TV, Thiagarajan RR, Rycus PT, Bartlett RH, Bratton SL. Extracorporeal membrane oxygenation in adults with severe respiratory failure: a multi-center database. Intensive Care Med. 2009;35:2105-14.

21. Chan T, Di Gennaro J, Farris RWD, Radman M, McMullan DM. Racial and ethnic variation in pediatric cardiac extracorporeal life support survival. Crit Care Med. 2017:45:670-8.

22. Sherwin ED, Gauvreau K, Scheurer MA, Rycus PT, Salvin JW, Almodovar MC, et al. Extracorporeal membrane oxygenation after stage 1 palliation for hypoplastic left heart syndrome. J Thorac Cardiovasc Surg. 2012;144:1337-43.

23. Friedland-Little JM, Aiyagari R, Yu S, Donohue JE, Hirsch-Romano JC. Survival through staged palliation: fate of infants supported by extracorporeal membrane oxygenation after the Norwood operation. Ann Thorac Surg. 2014; 97:659-65.

24. Cortes-Bergoderi M, Goel K, Murad MH, Allison T, Somers VK, Erwin PJ, et al. Cardiovascular mortality in Hispanics compared to non-Hispanic whites: a systematic review and meta-analysis of the Hispanic paradox. Eur J Intern Med. $2013 ; 24: 791-9$.
25. Medina-Inojosa J, Jean N, Cortes-Bergoderi M, Lopez-Jimenez F. The Hispanic paradox in cardiovascular disease and total mortality. Prog Cardiovasc Dis. 2014;57:286-92.

26. Chang RR, Rodriguez S, Lee M, Klitzner TS. Risk factors for deaths occurring within 30 days and 1 year after hospital discharge for cardiac surgery among pediatric patients. Am Heart J. 2006;152:386-93.

27. Gonzalez PC, Gauvreau K, Demone JA, Piercey GE, Jenkins KJ. Regional racia and ethnic differences in mortality for congenital heart surgery in children may reflect unequal access to care. Pediatr Cardiol. 2003;24:103-8.

28. Seifert HA, Howard DL, Silber JH, Jobes DR. Female gender increases the risk of death during hospitalization for pediatric cardiac surgery. J Thorac Cardiovasc Surg. 2007; 133:668-75.

29. Kucik JE, Nembhard WN, Donohue P, Devine O, Wang Y, Minkovitz CS, et al Community socioeconomic disadvantage and the survival of infants with congenital heart defects. Am J Public Health. 2014;104:e150-7.

30. Nembhard WN, Salemi JL, Ethen MK, Fixler DE, Dimaggio A, Canfield MA Racial/ethnic disparities in risk of early childhood mortality among children with congenital heart defects. Pediatrics. 2011;127:e1128-38.

31. Wang Y, Liu G, Druschel CM, Kirby RS. Maternal race/ethnicity and survival experience of children with congenital heart disease. J Pediatr. 2013;163: 1437-42.

32. Pasquali SK, Peterson ED, Jacobs JP, He X, Li JS, Jacobs ML, et al. Differentia case ascertainment in clinical registry versus administrative data and impact on outcomes assessment for pediatric cardiac operations. Ann Thorac Surg. 2013; 95:197-203.

33. Welke KF, Diggs BS, Karamlou T, Ungerleider RM. Comparison of pediatric cardiac surgical mortality rates from national administrative data to contemporary clinical standards. Ann Thorac Surg. 2009;87:216-22.

Key Words: extracorporeal membrane oxygenation, disparities, pediatric, congenital

Readers who found these articles interesting may also like to read the following papers found in recent and future issues of our sister publications, Seminars in Thoracic and Cardiovascular Surgery and Operative Techniques in Thoracic and Cardiovascular Surgery!

\section{Congenital: Mechanical Circulatory Support}

ORIGINAL SUBMISSION: Twenty-Five Year Outcomes of the Lateral Tunnel Fontan Procedure. Thomas G. Wilson. Semin Thoracic Surg 2017: 347-353.

Editorial Commentary: Excellence, Like Beauty, May Be in the Eyes of the Beholder. Ronald K. Woods. Semin Thoracic Surg 2017: 354-355.

ORIGINAL SUBMISSION: Unique Cannulation Technique and Atrioventricular Valve Excision for HeartWare HVAD in the Small Fontan Patient. Christopher E. Mascio. Oper Tech Thorac Cardiovasc 2016: 322-329.

ORIGINAL SUBMISSION: Mechanical Circulatory Support Following Norwood Palliation. Pirooz Eghtesady. Oper Tech Thorac Cardiovasc 2016: 330-338. 